\title{
A comparison of different solutions of the Bursa-Wolf model and of the 3D, 7-parameter datum transformation
}

\author{
József Závoti ${ }^{1} \cdot$ János Kalmár ${ }^{1}$
}

Received: 18 March 2015 / Accepted: 12 June 2015/Published online: 11 July 2015

(C) Akadémiai Kiadó 2015

\begin{abstract}
The present work deals with an important theoretical problem of geodesy: we are looking for a mathematical dependency between two spatial coordinate systems utilizing common pairs of points whose coordinates are given in both systems. In geodesy and photogrammetry the most often used procedure to move from one coordinate system to the other is the 3D, 7 parameter (Helmert) transformation. Up to recent times this task was solved either by iteration, or by applying the Bursa-Wolf model. Producers of GPS/GNSS receivers install these algorithms in their systems to achieve a quick processing of data. But nowadays algebraic methods of mathematics give closed form solutions of this problem, which require high level computer technology background. In everyday usage, the closed form solutions are much more simple and have a higher precision than earlier procedures and thus it can be predicted that these new solutions will find their place in the practice. The paper discusses various methods for calculating the scale factor and it also compares solutions based on quaternion with those that are based on rotation matrix making use of skew-symmetric matrix.
\end{abstract}

Keywords Quaternion-algebra - Bursa-Wolf model - Rotation matrix - Scale factor . 3D or 7-parameter datum (Helmert) transformation

\section{Introduction}

The conventional treatment of the 3D, 7-parameter datum transformation is given in Grafarend and Krumm (1995), in Grafarend and Kampmann (1996), and in Grafarend and Shan (1997). Subsequently Awange et al. (2004) has added extensions to the solutions.

József Závoti

zavoti@ggki.hu;

https://www.ggki.hu

János Kalmár

kalmar.janos@csfk.mta.hu

1 Research Centre for Astronomy and Earth Sciences, Hungarian Academy of Sciences,

Csatkai u. 6-8, Sopron 9400, Hungary 
Závoti (1999) has given a solution with limited conditions in L1 norm. In the application of computer algebraic systems to datum transformations, Awange and Grafarend (2002, 2003a, b, c) have taken new directions. In Hungary, Závoti (2005) gives the first algebraic solution to the problem, and his solution also proposes correction to the mathematical model. Závoti and Jancsó (2006) give the basic idea for bringing the problem to linear form, and this concept is described in more detail in Závoti (2012). Battha and Závoti (2009a, b) have applied computerized algebra to the problem of the intersection problem. Závoti and Fritsch (2011) have given a totally new solution for the outer orientation problem of photogrammetry. Horn (1987) is one of the earliest works to give a solution to the absolute orientation problem, but his solution is different from Závoti (2012). Závoti and Kalmár (2014) give a good summary of the differences between the two solutions.

\section{The model of the new solution for the 3D, 7-parameter similarity transformation}

Suppose that we have two distinct coordinate systems with $n$ common points given by their coordinates.

The 3D, 7-parameter (Helmert) overdetermined similarity transformation is given by the matrix equation:

$$
\boldsymbol{s}_{\mathbf{i}}=\boldsymbol{t}+\lambda \boldsymbol{R} \boldsymbol{p}_{\mathbf{i}}, \quad i=1,2, \ldots, n,
$$

where $\boldsymbol{s}_{\mathbf{i}}=\left[X_{i}, Y_{i}, Z_{i}\right]^{T}$ are the target coordinates, $\boldsymbol{t}=\left[X_{0}, Y_{0}, Z_{0}\right]^{T}$ is the unknown shift, $\lambda$ is the unknown scale-factor, $\boldsymbol{R}(\alpha, \beta, \gamma)$ is the rotation matrix, $\boldsymbol{p}_{\mathbf{i}}=\left[x_{i}, y_{i}, z_{i}\right]^{T}$ are the coordinates of the object points.

The $\boldsymbol{R}$ rotation matrix is parametrized using the three independent and unknown $\alpha, \beta$ and $\gamma$ Cardan-angles (Awange 2002), which belong to rotations about axes $z-y-x$ by the angle $\gamma, \beta$ and $\alpha$ consecutively.

$$
\boldsymbol{R}=\boldsymbol{R}_{1}(\alpha) \boldsymbol{R}_{2}(\beta) \boldsymbol{R}_{3}(\gamma) .
$$

Obviously, changing the order of the three rotations or the direction of the angles leads to rotation matrix.

The rotation angles can be obtained from the elements of the rotation matrix:

$$
\alpha=-\arctan \left(\frac{r_{23}}{r_{33}}\right), \beta=\arcsin \left(r_{13}\right), \gamma=-\arctan \left(\frac{r_{12}}{r_{11}}\right),
$$

where $r_{i j}$ is the j-th entry in the $\mathrm{i}$-th row of the matrix $\boldsymbol{R}$. Thus our goal is the determination of the rotation matrix.

Awange and Grafarend (2002) have introduced the (5) skew-symmetric matrix $\boldsymbol{C}^{\prime}$ that has the property:

$$
\boldsymbol{R}=\left(\boldsymbol{I}_{3}-\boldsymbol{C}^{\prime}\right)^{-1}\left(\boldsymbol{I}_{3}+\boldsymbol{C}^{\prime}\right)
$$

where $\boldsymbol{I}_{3}$ is the 3-dimesional identity matrix, and $\boldsymbol{C}^{\prime}$ is given by

$$
\boldsymbol{C}^{\prime}=\left[\begin{array}{ccc}
0 & -c & b \\
c & 0 & -a \\
-b & a & 0
\end{array}\right],
$$

with parameters $a, b$ and $c$. 
Multiplying from the left Eq. (1) with $\left(\boldsymbol{I}_{3}-\boldsymbol{C}^{\prime}\right)$, and applying Eq. (4), we obtain:

$$
\begin{aligned}
& {\left[\begin{array}{ccc}
1 & c & -b \\
-c & 1 & a \\
b & -a & 1
\end{array}\right]} \\
& \quad i=1,2, \ldots, n
\end{aligned}
$$

These equations are the basis of the algebraic solution of the 3D, 7-parameter (Helmert) transformation.

\section{Determination of the scale-factor of the 3D, 7-parameter similarity transformation}

Závoti (2012) has eliminated the shift parameters by reducing the coordinates to the center of gravity. He also has shown that during the solution of the overdetermined system of equations parameters $a, b$ and $c$ are eliminated, and the following overdetermined system of equations, quadratic in the unknown $\lambda$ parameter, are obtained:

$$
\lambda^{2}\left(x_{i s}^{2}+y_{i s}^{2}+z_{i s}^{2}\right)=X_{i s}^{2}+Y_{i s}^{2}+Z_{i s}^{2}, \quad i=1,2, \ldots, n,
$$

where $\quad X_{i s}=X_{i}-X_{s}, Y_{i s}=Y_{i}-Y_{s}, Z_{i s}=Z_{i}-Z_{s} i=1,2, \ldots, n, \quad x_{i s}=x_{i}-x_{s}, y_{i s}=$ $y_{i}-y_{s}, z_{i s}=z_{i}-z_{s}, \quad i=1,2, \ldots, n,\left(X_{s}, Y_{s}, Z_{s}\right),\left(x_{s}, y_{s}, z_{s}\right)$ are coordinates the centre of gravity.

The system of Eq. (7) is overdetermined, with several solutions according to a chosen error function.

\subsection{Solution I}

The (7) system of equations is written as a product:

$$
\begin{aligned}
& \left(\lambda \sqrt{x_{i s}^{2}+y_{i s}^{2}+z_{i s}^{2}}-\sqrt{X_{i s}^{2}+Y_{i s}^{2}+Z_{i s}^{2}}\right)\left(\lambda \sqrt{x_{i s}^{2}+y_{i s}^{2}+z_{i s}^{2}}+\sqrt{X_{i s}^{2}+Y_{i s}^{2}+Z_{i s}^{2}}\right)=0, \\
& i=1,2, \ldots, n
\end{aligned}
$$

Let us consider the first factor in the above product. The system of equations to be solved is:

$$
\lambda \sqrt{x_{i s}^{2}+y_{i s}^{2}+z_{i s}^{2}}=\sqrt{X_{i s}^{2}+Y_{i s}^{2}+Z_{i s}^{2}}, \quad i=1,2, \ldots, n .
$$

Add up all these equations! The solution for $\lambda$ of the overdetermined system of equations - taking into account that only the positive root has a physical meaning for us-is given, according to Závoti (2012), by the following well known equation:

$$
\lambda_{1}=\frac{\sum_{i=1}^{n} \sqrt{X_{i s}^{2}+Y_{i s}^{2}+Z_{i s}^{2}}}{\sum_{i=1}^{n} \sqrt{x_{i s}^{2}+y_{i s}^{2}+z_{i s}^{2}}} .
$$


Albertz and Kreiling (1975) have shown that the $\lambda$ scale-factor can be obtained as the quotient of the sums of the coordinates of the points, taken in the coordinate system with origin in the center of gravity. Thus we have transformed the quadratic Eq. (7) to linear equations, as opposed to the procedure given in Awange and Grafarend (2002), which requires the cumbersome separation of the roots of a polynomial of degree 4 .

\subsection{Solution II}

Adding up all equations in (7) gives

$$
\lambda^{2} \sum_{i=1}^{n}\left(x_{i s}^{2}+y_{i s}^{2}+z_{i s}^{2}\right)=\sum_{i=1}^{n}\left(X_{i s}^{2}+Y_{i s}^{2}+Z_{i s}^{2}\right) .
$$

There is a simple solution of this equation in non-negative real numbers without transforming it to a product. After taking into account that a root with physical meaning must be positive, we obtain for the lambda scale-factor the following equation, given in Horn (1987) using quaternions, which is also the solution of the Bursa-Wolf model:

$$
\lambda_{2}=\sqrt{\frac{\sum_{i=1}^{n}\left(X_{i s}^{2}+Y_{i s}^{2}+Z_{i s}^{2}\right)}{\sum_{i=1}^{n}\left(x_{i s}^{2}+y_{i s}^{2}+z_{i s}^{2}\right)} .}
$$

Thus we can obtain the unique $\lambda$ scale-factor from the quadratic equations as opposed to the complicated separation procedure of the roots of a polynomial of degree 4 given by Awange and Grafarend (2002).

\subsection{Solution III}

Our starting point is again the system of Eq. (7). We want to obtain a least squares solution for $\lambda$ using intermediary equations. Elementary steps, given in full detail in (23)-(26), yield:

$$
\lambda_{3}=\frac{\sum_{i=1}^{n} \sqrt{\left(x_{i s}^{2}+y_{i s}^{2}+z_{i s}^{2}\right)\left(X_{i s}^{2}+Y_{i s}^{2}+Z_{i s}^{2}\right)}}{\sum_{i=1}^{n}\left(x_{i s}^{2}+y_{i s}^{2}+z_{i s}^{2}\right)} .
$$

Thus there are three different solution procedures (estimations) for the $\lambda$ scale factor of the 3D, 7-parameter (Helmert) transformation.

\section{Determination of the linear and shift parameters}

After having determined the scale-factor, the problem can be written in linear form, and the adjustment model for the linear problem can be given. This procedure makes it possible to include arbitrarily many equations (common points), and give a solution for the parameters $a, b$, and $c$.

Závoti (2013) has determined the normal matrix of the problem: 


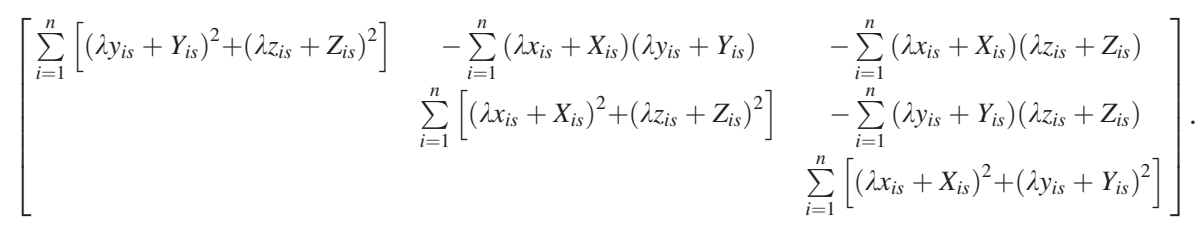

(The symmetric entries of the normal matrix are not listed).

The normal vector is obtained in a similar way:

$$
2 \lambda\left[\begin{array}{c}
\sum_{i=1}^{n}\left(y_{i s} Z_{i s}-z_{i s} Y_{i s}\right) \\
\sum_{i=1}^{n}\left(z_{i s} X_{i s}-x_{i s} Z_{i s}\right) \\
\sum_{i=1}^{n}\left(x_{i s} Y_{i s}-y_{i s} X_{i s}\right)
\end{array}\right]
$$

There are several procedures to obtain the parameters $a, b$ and $c$ from the $3 \times 3$ normal system of equations, we have chosen the singular value decomposition because of its stability. Utilizing special properties of the normal matrix the rotation parameters in (3) can also be determined.

The shift parameters $X_{0}, Y_{0}$ and $Z_{0}$ can be determined after Eq. (1) is rewritten to a system with the center of gravity as origin

$$
\left[\begin{array}{l}
X_{0} \\
Y_{0} \\
Z_{0}
\end{array}\right]=\left[\begin{array}{c}
X_{s} \\
Y_{s} \\
Z_{s}
\end{array}\right]-\lambda R\left[\begin{array}{l}
x_{s} \\
y_{s} \\
z_{s}
\end{array}\right] .
$$

Parameters for the precision, variance and covariance are computed in the conventional way.

\section{The Bursa-Wolf model of the datum-transformation}

Equation (13) can also be obtained in the following way ( $\overline{\boldsymbol{s}}$ and $\overline{\boldsymbol{p}}$ are the centers of gravity in the two systems):

$$
\begin{array}{r}
\Delta \boldsymbol{s}_{i}=\boldsymbol{s}_{i}-\overline{\boldsymbol{s}} \Rightarrow \boldsymbol{s}_{i}=\Delta \boldsymbol{s}_{i}+\overline{\boldsymbol{s}} \\
\Delta \boldsymbol{p}_{i}=\boldsymbol{p}_{i}-\overline{\boldsymbol{p}} \Rightarrow \boldsymbol{p}_{i}=\Delta \boldsymbol{p}_{i}+\overline{\boldsymbol{p}} .
\end{array}
$$

Substituting this into the transformation Eq. (1) gives:

$$
\Delta \boldsymbol{s}_{i}+\overline{\boldsymbol{s}}=\boldsymbol{t}+\lambda \cdot \boldsymbol{R} \cdot\left(\Delta \boldsymbol{p}_{i}+\overline{\boldsymbol{p}}\right), \quad i=1,2, \ldots, n .
$$

Reordering terms gives:

$$
\Delta \boldsymbol{s}_{i}+\overline{\boldsymbol{s}}=\boldsymbol{t}+\lambda \cdot \boldsymbol{R} \cdot \overline{\boldsymbol{p}}+\lambda \cdot \boldsymbol{R} \cdot \Delta \boldsymbol{p}_{i}, \quad i=1,2, \ldots, n .
$$

Since (1) holds for the centers of gravity $\overline{\boldsymbol{s}}$ and $\overline{\boldsymbol{p}}$ too, so the term in the middle of (19) can be omitted and thus we are left with:

$$
\Delta \boldsymbol{s}_{i}=\lambda \cdot \boldsymbol{R} \cdot \Delta \boldsymbol{p}_{i}, \quad i=1,2, \ldots, n .
$$


Having temporarily eliminated the unknown shift vector $\boldsymbol{t}$ we are left with $\lambda$ and $\boldsymbol{R}$.

Based on Eq. (1) the shift vector $t$ in the Bursa-Wolf model is obtained by taking averages:

$$
\boldsymbol{t}=\sum_{i} \frac{\boldsymbol{s}_{i}-\lambda \cdot \boldsymbol{R} \cdot \boldsymbol{p}_{i}}{n}=\sum_{i} \frac{\boldsymbol{s}_{i}}{n}-\lambda \cdot \boldsymbol{R} \sum_{i} \frac{\boldsymbol{p}_{i}}{n}=\overline{\boldsymbol{s}}-\lambda \cdot \boldsymbol{R} \cdot \overline{\boldsymbol{p}},
$$

so (21) and (16) are equivalent.

We now want to deal with the scale-factor. In order to make the comparison more simple, we rewrite Eq. (10) using the notations of:

$$
\lambda_{1}=\sum_{i=1}^{n} \sqrt{\Delta \boldsymbol{s}_{i}^{T} \cdot \Delta \boldsymbol{s}_{i}} / \sum_{i=1}^{n} \sqrt{\Delta \boldsymbol{p}_{i}^{T} \cdot \Delta \boldsymbol{p}_{i}} .
$$

The scale factor $\lambda$ in (10) and (12) seems to be similar, but there is an important difference: in (10) taking square-root comes first and then follows the summing up, while in (12) the order is reversed. On the other hand, both (12) and (10) are statistical estimates of the scale-factor (their difference originates from the error equations), but in error-free case (10) and (12) give the same results. In fact, in the case of the ideal error-free Helmert transformation, the quotient of every distance and its image is constant $(\lambda)$-and this is also true for the coordinates relative to the center of gravity-since the center of gravity is replaced during the transformation, and thus the distances from the center of gravity can be obtained from the coordinates relative to the center of gravity:

$$
\sqrt{\Delta \boldsymbol{s}_{i}^{T} \cdot \Delta \boldsymbol{s}_{i}}, \sqrt{\Delta \boldsymbol{p}_{i}^{T} \cdot \Delta \boldsymbol{p}_{i}}, \quad i=1,2, \ldots, n .
$$

In the error-free case the dependency between the distances can be given by making use of the scale-factor:

$$
\sqrt{\Delta s_{i}^{T} \cdot \Delta s_{i}}=\lambda \cdot \sqrt{\Delta p_{i}^{T} \cdot \Delta p_{i}}, \quad i=1,2, \ldots, n .
$$

Now we can verify that substituting (24) into (10) and into (12) leads to equality $\lambda=\lambda_{1}=\lambda_{2}$, and thus the two statistical estimates (the theoretical scale) is identical.

From (24) we obtain the error-equations:

$$
v_{i}=\sqrt{\Delta \boldsymbol{s}_{i}^{T} \cdot \Delta \boldsymbol{s}_{i}}-\lambda \cdot \sqrt{\Delta \boldsymbol{p}_{i}^{T} \cdot \Delta \boldsymbol{p}_{i}}, \quad i=1,2, \ldots n,
$$

and these yield the following statistical estimates, which are different from (10) and (12), but have the same results in error-free case:

$$
\lambda=\sum_{i=1}^{n} \sqrt{\left(\Delta \boldsymbol{s}_{i}^{T} \cdot \Delta \boldsymbol{s}_{i}\right) \cdot\left(\Delta \boldsymbol{p}_{i}^{T} \cdot \Delta \boldsymbol{p}_{i}\right)} / \sum_{i=1}^{n} \Delta \boldsymbol{p}_{i}^{T} \cdot \Delta \boldsymbol{p}_{i} .
$$

In the error-free case (22) and (26) are identical estimators of $\lambda$, so it gives:

$$
\sum_{i=1}^{n} \sqrt{\Delta \boldsymbol{s}_{i}^{T} \cdot \Delta \boldsymbol{s}_{i}} / \sum_{i=1}^{n} \sqrt{\Delta \boldsymbol{p}_{i}^{T} \cdot \Delta \boldsymbol{p}_{i}}=\sum_{i=1}^{n} \sqrt{\left(\Delta \boldsymbol{s}_{i}^{T} \cdot \Delta \boldsymbol{s}_{i}\right) \cdot\left(\Delta \boldsymbol{p}_{i}^{T} \cdot \Delta \boldsymbol{p}_{i}\right)} / \sum_{i=1}^{n} \Delta \boldsymbol{p}_{i}^{T} \cdot \Delta \boldsymbol{p}_{i} .
$$




\section{Determination of the unknowns from a minimax-problem}

We consider the residual vectors of (20):

$$
\Delta v_{i}=\Delta \boldsymbol{s}_{i}-\lambda \cdot \boldsymbol{R} \cdot \Delta \boldsymbol{p}_{i}, \quad i=1,2, \ldots, n
$$

We consider the following optimum-problem:

$$
\min _{\lambda, \boldsymbol{R}} \sum_{i} \Delta v_{i}^{T} \cdot \Delta v_{i}=\min _{\lambda, \boldsymbol{R}} \sum_{i}\left(\Delta \boldsymbol{s}_{i}-\lambda \cdot \boldsymbol{R} \cdot \Delta \boldsymbol{p}_{i}\right)^{T} \cdot\left(\Delta \boldsymbol{s}_{i}-\lambda \cdot \boldsymbol{R} \cdot \Delta \boldsymbol{p}_{i}\right) .
$$

Since $\boldsymbol{R}$ is an orthogonal matrix $\left(\boldsymbol{R}^{T} \boldsymbol{R}=\boldsymbol{I}_{3}\right)$ we can rewrite it as follows:

$$
\min _{\lambda, \boldsymbol{R}}\left\{\sum_{i}\left(\Delta \boldsymbol{s}_{i}^{T} \cdot \Delta \boldsymbol{s}_{i}\right)-2 \lambda\left(\sum_{i} \Delta \boldsymbol{s}_{i}^{T} \cdot \boldsymbol{R} \cdot \Delta \boldsymbol{p}_{i}\right)+\lambda^{2} \sum_{i}\left(\Delta \boldsymbol{p}_{i}^{T} \cdot \Delta \boldsymbol{p}_{i}\right)\right\} .
$$

Minimax value is obtained where the partial derivative for $\lambda$ is zero:

$$
\lambda=\sum_{i}\left(\Delta \boldsymbol{s}_{i}^{T} \cdot \boldsymbol{R} \cdot \Delta \boldsymbol{p}_{i}\right) / \sum_{i}\left(\Delta \boldsymbol{p}_{i}^{T} \cdot \Delta \boldsymbol{p}_{i}\right) .
$$

By (20) can be written

$$
\frac{1}{\lambda} \Delta \boldsymbol{s}_{i}=\boldsymbol{R} \cdot \Delta \boldsymbol{p}_{i}, \quad i=1,2, \ldots, n .
$$

We substitute Eq. (32) into Eq. (31):

$$
\lambda=\frac{1}{\lambda} \sum_{i}\left(\Delta \boldsymbol{s}_{i}^{T} \cdot \Delta \boldsymbol{s}_{i}\right) / \sum_{i}\left(\Delta \boldsymbol{p}_{i}^{T} \cdot \Delta \boldsymbol{p}_{i}\right),
$$

which yields the well known Horn-Eq. (12):

$$
\lambda=\sqrt{\sum_{i}\left(\Delta \boldsymbol{s}_{i}^{T} \cdot \Delta \boldsymbol{s}_{i}\right) / \sum_{i}\left(\Delta \boldsymbol{p}_{i}^{T} \cdot \Delta \boldsymbol{p}_{i}\right)} .
$$

Since $\lambda$ is already known the minimax value for (30) depends only on the $\boldsymbol{R}$ rotation matrix, and thus the first and third (constant) sums in (30) can be omitted, but from the second the maximum is obtained since there is a change of sign. The denominator can be omitted since it is always positive, and thus we are left with:

$$
\max _{\boldsymbol{R}} \sum_{i}\left(\Delta \boldsymbol{s}_{i}^{T} \cdot \boldsymbol{R} \cdot \Delta \boldsymbol{p}_{i}\right)
$$

\section{The solution of the minimax problem using quaternion-algebra}

The basic equations for quaternions are: 


$$
\begin{gathered}
\underline{\boldsymbol{q}}=q_{0}+q_{1} \cdot \boldsymbol{i}+q_{2} \cdot \boldsymbol{j}+q_{3} \cdot \boldsymbol{k}=q_{0}+\boldsymbol{q}, \\
\underline{\boldsymbol{q}}^{*}=q_{0}-\boldsymbol{q}=\left(q_{0},-\boldsymbol{q}^{T}\right)^{T} \quad\left(\underline{\boldsymbol{q}}^{*} \text { is the conjugate of } \underline{\boldsymbol{q}}\right), \\
\|\underline{\boldsymbol{q}}\|=\sqrt{q_{0}^{2}+q_{1}^{2}+q_{2}^{2}+q_{3}^{2}} \quad(\|\underline{\boldsymbol{q}}\| \text { is the length of } \underline{\boldsymbol{q}}), \\
\boldsymbol{C}(\boldsymbol{q})=\left[\begin{array}{ccc}
0 & -q_{3} & q_{2} \\
q_{3} & 0 & -q_{1} \\
-q_{2} & q_{1} & 0
\end{array}\right], \\
\boldsymbol{Q}^{+}=\left[\begin{array}{ccc}
q_{0} & -\boldsymbol{q}^{T} \\
\boldsymbol{q} & q_{0} \cdot \boldsymbol{I}_{3}+C(\boldsymbol{q})
\end{array}\right], \quad \boldsymbol{Q}^{-}=\left[\begin{array}{cc}
q_{0} & -\boldsymbol{q}^{T} \\
\boldsymbol{q} & q_{0} \cdot \boldsymbol{I}_{3}-C(\boldsymbol{q})
\end{array}\right] .
\end{gathered}
$$

Using quaternions (4-dimensional vectors $\left.\underline{\boldsymbol{s}}=\left(0, \Delta \boldsymbol{s}^{T}\right)^{T}, \boldsymbol{p}=\left(0, \Delta \boldsymbol{p}^{T}\right)^{T}\right)$, the bilinear form (35) can be rewritten so that the rotation matrix $\boldsymbol{R}$ is replaced with the unknown quaternion $\underline{\boldsymbol{q}}=\left(q_{0}, \boldsymbol{q}^{T}\right)^{T}$. Shen et al. (2006) give the following equation for the rotation matrix $\boldsymbol{R}$ and the $\underline{q}$ quaternion:

$$
\boldsymbol{R}=\left(q_{0}^{2}-\boldsymbol{q}^{T} \cdot \boldsymbol{q}\right) \cdot I_{3}+2\left(\boldsymbol{q} \cdot \boldsymbol{q}^{T}+q_{0} \cdot \boldsymbol{C}(\boldsymbol{q})\right) .
$$

Now we can rewrite (35):

$$
\max _{\boldsymbol{R}} \sum_{i}\left(\Delta \boldsymbol{s}_{i}^{T} \cdot \boldsymbol{R} \cdot \Delta \boldsymbol{p}_{i}\right)=\max _{\underline{q}} \sum_{i}\left(\underline{s}_{i}^{T} \cdot \boldsymbol{Q}^{+} \cdot \boldsymbol{P}_{i}^{+} \cdot \underline{\boldsymbol{q}}^{*}\right)=\max _{\underline{q}} \underline{\boldsymbol{q}}^{T} \cdot \boldsymbol{N} \cdot \underline{\boldsymbol{q}}
$$

where the $4 \times 4$ matrix $N$ is given by

$$
N=\sum_{i}\left[\begin{array}{cc}
\Delta s_{i}^{T} \cdot \Delta p_{i} & \Delta s_{i}^{T} \cdot C\left(\Delta p_{i}\right) \\
-C\left(\Delta s_{i}\right) \cdot \Delta p_{i} & \Delta s_{i} \cdot \Delta p_{i}^{T}+C\left(\Delta s_{i}\right) \cdot C\left(\Delta p_{i}\right)
\end{array}\right]
$$

The maximum of the quadratic form (38) is obtained when $\underline{\boldsymbol{q}}$ is an eigenvector of $\boldsymbol{N}$, and then its value is equal to the eigenvalue of $N$ (Shen et al. 2006), and thus we have to determine the maximal eigenvalue of $\boldsymbol{N}$ and the eigenvector $\boldsymbol{q}$ (the quaternion we are looking for) belonging to this eigenvalue.

Using the quaternion $\underline{\boldsymbol{q}}$, we obtain the rotation matrix $\boldsymbol{R}=\left(r_{i j}\right)$ from (37), and the rotation angles from (3). The shift vector $\boldsymbol{t}$ is then obtained from (21) by taking averages.

\section{Connection between the parameters of the rotation matrix $R$ in formulas (4) and (37)}

The skew-symmetric matrix $\boldsymbol{C}^{\prime}$ describes the rotation matrix $\boldsymbol{R}$ of the nonlinear similarity transformation according to (5), the $\boldsymbol{q}$ quaternion describes it according to (36). First we rewrite the rotation matrix $\boldsymbol{R}$ from (5):

$$
\boldsymbol{R}=\frac{1}{1+a^{2}+b^{2}+c^{2}}\left[\begin{array}{ccc}
1+a^{2}-b^{2}-c^{2} & 2(a b-c) & 2(a c+b) \\
2(a b+c) & 1-a^{2}+b^{2}-c^{2} & 2(b c-a) \\
2(a c-b) & 2(b c+a) & 1-a^{2}-b^{2}+c^{2}
\end{array}\right]
$$


Then the components of the quaternion $\underline{q}$ are used to obtain the rotation matrix from (36) and (37):

$$
\boldsymbol{R}=\left[\begin{array}{ccc}
q_{0}^{2}+q_{1}^{2}-q_{2}^{2}-q_{3}^{2} & 2\left(q_{1} q_{2}-q_{0} q_{3}\right) & 2\left(q_{1} q_{3}+q_{0} q_{2}\right) \\
2\left(q_{1} q_{2}+q_{0} q_{3}\right) & q_{0}^{2}-q_{1}^{2}+q_{2}^{2}-q_{3}^{2} & 2\left(q_{2} q_{3}-q_{0} q_{1}\right) \\
2\left(q_{1} q_{3}-q_{0} q_{2}\right) & 2\left(q_{2} q_{3}+q_{0} q_{1}\right) & q_{0}^{2}-q_{1}^{2}-q_{2}^{2}+q_{3}^{2}
\end{array}\right]
$$

One may ask, in what cases are the rotation matrices obtained from (40) and from (41) identical?

Let

$$
a=\frac{q_{1}}{q_{0}}, b=\frac{q_{2}}{q_{0}}, c=\frac{q_{3}}{q_{0}} .
$$

Let us substitute parameters $a, b$ and $c$ from (42) into (40):

$$
\boldsymbol{R}=\frac{q_{0}^{2}}{q_{0}^{2}+q_{1}^{2}+q_{2}^{2}+q_{3}^{2}}\left[\begin{array}{ccc}
\frac{q_{0}^{2}+q_{1}^{2}-q_{2}^{2}-q_{3}^{2}}{q_{0}^{2}} & 2 \frac{q_{1} q_{2}-q_{0} q_{3}}{q_{0}^{2}} & 2 \frac{q_{1} q_{3}+q_{0} q_{2}}{q_{0}^{2}} \\
2 \frac{q_{1} q_{2}+q_{0} q_{3}}{q_{0}^{2}} & \frac{q_{0}^{2}-q_{1}^{2}+q_{2}^{2}-q_{3}^{2}}{q_{0}^{2}} & 2 \frac{q_{2} q_{3}-q_{0} q_{1}}{q_{0}^{2}} \\
2 \frac{q_{1} q_{3}-q_{0} q_{2}}{q_{0}^{2}} & 2 \frac{q_{2} q_{3}+q_{0} q_{1}}{q_{0}^{2}} & \frac{q_{0}^{2}-q_{1}^{2}-q_{2}^{2}+q_{3}^{2}}{q_{0}^{2}}
\end{array}\right] .
$$

We can simplify this by omitting $q_{0}^{2}$ from both the scalar factor of the matrix and the denominators in the entries of the matrix, and by taking into account the equality $q_{0}^{2}+q_{1}^{2}+q_{2}^{2}+q_{3}^{2}=1$, and then we obtain an equation identical to (41). Thus we have derived (41) from (40).

Now let

$$
q_{1}=q_{0} a, q_{2}=q_{0} b, q_{3}=q_{0} c .
$$

Then

$$
1=q_{0}^{2}+q_{1}^{2}+q_{2}^{2}+q_{3}^{2}=q_{0}^{2}\left(1+a^{2}+b^{2}+c^{2}\right),
$$

leads to the equality

$$
q_{0}= \pm \frac{1}{\sqrt{1+a^{2}+b^{2}+c^{2}}} .
$$

We now substitute (44) and (46) into (41), and obtain the following equation for the rotation matrix $\boldsymbol{R}$ :

$$
\boldsymbol{R}=\left[\begin{array}{ccc}
\frac{1+a^{2}-b^{2}-c^{2}}{1+a^{2}+b^{2}+c^{2}} & 2 \frac{a b-c}{1+a^{2}+b^{2}+c^{2}} & 2 \frac{a c+b}{1+a^{2}+b^{2}+c^{2}} \\
2 \frac{a b+c}{1+a^{2}+b^{2}+c^{2}} & \frac{1-a^{2}+b^{2}-c^{2}}{1+a^{2}+b^{2}+c^{2}} & 2 \frac{b c-a}{1+a^{2}+b^{2}+c^{2}} \\
2 \frac{a c-b}{1+a^{2}+b^{2}+c^{2}} & 2 \frac{b c+a}{1+a^{2}+b^{2}+c^{2}} & \frac{1-a^{2}-b^{2}+c^{2}}{1+a^{2}+b^{2}+c^{2}}
\end{array}\right]
$$


Table 1 Relation between quaternions and $a, b$, $c$ parameters

$$
\begin{array}{lrl}
\hline q_{0} & =\frac{1}{\sqrt{1+a^{2}+b^{2}+c^{2}}} & a=\frac{q_{1}}{q_{0}} \\
q_{1}=q_{0} a & b=\frac{q_{2}}{q_{0}} \\
q_{2}=q_{0} b & c=\frac{q_{3}}{q_{0}}
\end{array}
$$

which is identical to (40). We can summarize our result: the connection between the quaternion components $q_{0}, q_{1}, q_{2}$ and $q_{3}$ of the Bursa-Wolf model and the parameters $a, b$ and $c$ of the skew symmetric matrix $C^{\prime}$ is described by the equations in Table 1.

\section{Summary}

In the present work we have given a general model for the 3D, 7-parameter (Helmert) similarity transformation, which can be used to obtain several different solutions, and which includes as a special case the Bursa-Wolf model. In our method we introduce an overdetermined system of equations for the scale-factor, which is then solved using different principles. The advantage of our model is that the determination of the scale-factor makes it possible to obtain the solution for the original nonlinear problem from the solution of a linear problem. We have given a new least-squares solution for the scale-factor, which is numerically identical to the relevant parameter of the Bursa-Wolf model.

We have shown that there is a functional connection between the quaternions of the Bursa-Wolf model and the entries of the skew-symmetric matrix given by Awange and Grafarend.

\section{Appendix: A numerical example for different solutions of the 3D, 7-parameter transformation}

We are considering the example in Awange and Grafarend (2002), which was also used in Závoti (2013) (Table 2). The origins of the two coordinate systems are given in VGS84 and in a local system. To verify the numerical computations, we have written a MATLAB program, which allows a choice between solutions I, II, and III (see chapter 3, formula (10), (12), (13)) for the scale-factor. Following the determination of the scale-factor, our procedure uses in all three cases our linear model. Thus the rotation and shift parameters of the Bursa-Wolf model are also determined from the linear model. The equivalence of the two solutions II and III has already been proved in Papp (2013) and Závoti (2013). The rotation angles $\alpha, \beta$ and $\gamma$ can be obtained from the $\boldsymbol{R}$ rotation matrix (40) using Eq. (3). It can be seen that there is no need to give a starting value, the equations have not to be expanded, there is no need for iteration, and the procedure can be used for arbitrary rotation angles. The results for the solution of the nonlinear problem using the algorithms of the present work are given in Table 3.

Both methods yield, within computational precision, the following identical values for the Cardan-angles:

$$
\alpha=-0.9984976709 \text { ['] } \beta=-0.8936957645 \text { ['] } \gamma=0.9930877298 \text { ['] }
$$

Because solutions II and III yield the same numerical values, these common values are only listed once in the header of Table 3 under the entry Bursa-Wolf. 
Table 2 Coordinates for local system and WGS-84 system

\begin{tabular}{lllllll}
\hline $\mathrm{Nr}$ & $X_{i}$ & $Y_{i}$ & $Z_{i}$ & $x_{i}$ & $y_{i}$ & $z_{i}$ \\
\hline 1 & 4157870.237 & 664818.678 & 4775416.524 & 4157222.543 & 664789.307 & 4774952.099 \\
2 & 4149691.049 & 688865.785 & 4779096.588 & 4149043.336 & 688836.443 & 4778632.188 \\
3 & 4173451.354 & 690369.375 & 4758594.075 & 4172803.511 & 690340.078 & 4758129.701 \\
4 & 4177796.064 & 643026.700 & 4761228.899 & 4177148.376 & 642997.635 & 4760764.800 \\
5 & 4137659.549 & 671837.337 & 4791592.531 & 4137012.190 & 671808.029 & 4791128.215 \\
6 & 4146940.228 & 666982.151 & 4784324.099 & 4146292.729 & 666952.887 & 4783859.856 \\
7 & 4139407.506 & 702700.227 & 4786016.645 & 4138759.902 & 702670.738 & 4785552.196 \\
\hline
\end{tabular}

Table 3 Numerical results

\begin{tabular}{lclc}
\hline Unknowns & I. Solution (Závoti) & Unknowns & II-III. Solutions (Bursa-Wolf) \\
\hline$\lambda$ & 1.0000047879 & $\lambda$ & 1.0000055825 \\
& & $q_{0}$ & 0.9999999999 \\
$a$ & 0.0000024204 & $q_{1}$ & 0.0000024204 \\
$b$ & -0.0000021664 & $q_{2}$ & -0.0000021664 \\
$c$ & -0.0000024073 & $q_{3}$ & -0.0000024073 \\
$X_{0}$ & 645.1812 & $t_{x}$ & 641.8804 \\
$Y_{0}$ & 69.1921 & $t_{y}$ & 68.6553 \\
$Z_{0}$ & 420.1933 & $t_{z}$ & 416.3981 \\
$\sigma_{0}$ & 0.0786340816 & $\sigma_{0}$ & 0.0772336608 \\
\hline
\end{tabular}

We note that the quaternions $q_{1}, q_{2}$ and $q_{3}$, and the parameters $a, b$, and $c$ of the skewsymmetric matrix $C^{\prime}$ are equal only within computational precision. Differences in subsequent digits follow from Eq. (42). Greater differences exist between the scale-factors $\lambda$ and the shift parameters of the two methods. The differences in the scale-factors are a consequence of the differences between Eqs. (10) and (12), and this may result in the relative differences in the shift parameters. The Bursa-Wolf model makes the measurement errors minimal by an exclusive use of least squares methods, while our model can also be applied not only in least squares cases.

\section{References}

Albertz J, Kreiling W (1975) Photogrammetric guide. Herbert Wichmann Verl, Karlsruhe, pp 58-60

Awange JL (2002) Gröbner bases, multipolynomial resultants and the Gauss-Jacobbi combinatorical algorithms-adjustment of nonlinear GPS/LPS observations. Dissertation, Geodätisches Institut der Universität Stuttgart

Awange JL, Grafarend EW (2002) Linearized least squares and nonlinear Gauss-Jacobbi combinatorical algorithm applied to the 7 parameter datum transformation $c_{7}(3)$ problem. Zeitschrift für Vermessungswesen 127:109-116

Awange JL, Grafarend EW (2003a) Closed form solution of the overdetermined nonlinear 7 parameter datum transformatiotn. Allgemeine Vermessungsnachrichten 110:130-149

Awange JL, Grafarend EW (2003b) Explicit solution of the overdetermined three-dimensional resection problem. J Geod 76:605-616 
Awange JL, Grafarend EW (2003c) Polinomial Optimization of the 7-parameter datum transformation problem when only three stations in both system are given. Zeitschrift für Vermessungswesen 128:266-270

Awange JL, Grafarend EW, Fukuda Y (2004) Exact solution of the nonlinear 7-parameter datum trsformaton by Groebner basis. Bul. di Geodesia e Scienze Affini 63:117-127

Battha L, Závoti J (2009a) Solution of the intersection problem by the Sylvester-resultant and a comparison of two solutions of the 2D similarity transformation. Acta Geod Geoph Hung 44(4):429-438

Battha L, Závoti J (2009b) The intersection problem and the 2D similarity transformation. Publ Geomat 12:19-26

Grafarend EW, Kampmann $\mathrm{G}(1996) \mathrm{C}_{10}(3)$ : the ten parameter conformal group as a datum transformation in threedimensional Euclidean space. Zeitschrift für Vermessungswesen 121:68-77

Grafarend EW, Krumm F (1995) Curvilinear geodetic datum transformations. Zeitschrift für Vermessungswesen 120:334-350

Grafarend EW, Shan J (1997) Estimable quantities in projective networks. Zeitschrift für Vermessungswesen 122:323-333

Horn BKP (1987) Closed form solution of absolute orientation using unit quaternions. J Optical Soc Am 4:629-642

Papp E (2013) Geodetic datum transformation by quaternion. Publ Geomat 16:17-28

Shen YZ, Chen Y, Zheng DH (2006) A quaternion-based geodetic datum transformation algorithm. J Geod 80:233-239

Závoti J (1999) Up to date mathematical methods in geodesy. Publ Geomat 2:149

Závoti J (2005) The closed form soltion of 7 parameter 3D transformation. Publ Geomat 8:53-60

Závoti J (2012) A simple proof of the solutions of the Helmert- and the overdetermined nonlinear 7-parameter datum transformation. Acta Geod Geoph Hung 47(4):453-464

Závoti J (2013) New treatment of the solution of 2D and 3D non-linear similarity (Helmert) transformations. Publ Geomat 16:7-16

Závoti J, Fritsch D (2011) A first attempt at a new algebraic solution of the exterior orientation of photogrammetry. Acta Geod Geoph Hung 46:317-325

Závoti J, Jancsó T (2006) The solution of the 7-parameter datum transformation problem with-and without the Gröbner basis. Acta Geod Geoph Hung 41(1):87-100

Závoti J, Kalmár J (2014) Several alternative possibilities for the solution of 3D non-linear similarity datum transformation compared to the Bursa-Wolf model. Publ Geomat 17:7-18 